\title{
Application of Hydroxyporphyrins-Based Phosphite-Type Ligands to Asymmetric Pd-Catalyzed Allylic Substitution Reactions
}

\author{
Konstantin N. Gavrilov, ${ }^{a}{ }^{\circledR}$ Sergey V. Zheglov, ${ }^{a}$ Marina G. Maksimova, ${ }^{a}$

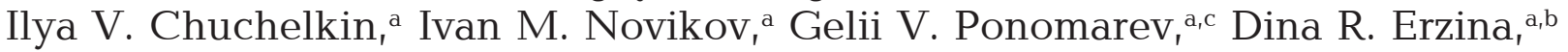 \\ and Igor S. Mikhel ${ }^{a, b}$
}

\section{a S.A. Esenin Ryazan State University, 390000 Ryazan, Russian Federation}

${ }^{\mathrm{b}}$ A.N. Frumkin Institute of Physical Chemistry and Electrochemistry, Russian Academy of Sciences, 119071 Moscow, Russian Federation

${ }^{\mathrm{c}}$ V.N. Orekhovich Institute of Biomedical Chemistry, 119121 Moscow, Russian Federation

${ }^{\circledR}$ Corresponding authorE-mail: k.gavrilov@rsu.edu.ru

\begin{abstract}
Diamidophosphite and phosphite ligands with (S)-2-(anilinomethyl)pyrrolidine or (S)-BINOL backbone and porphyrin cores were obtained. The use of these ligands provides up to $92 \%$ ee (enantiomeric excess) in Pd-catalyzed asymmetric alkylation of (E)-1,3-diphenylallyl acetate with dimethyl malonate, up to $82 \%$ ee in its sulfonylation with sodium p-toluene sulfinate and up to $76 \%$ ee in its amination with pyrrolidine. Also, up to $90 \%$ ee was achieved in Pdcatalyzed allylic alkylation of cinnamyl acetate with ethyl 2-oxocyclohexane-1-carboxylate. The influence of the structural moieties such as the nature of phosphorus-containing ring or porphyrin exocyclic substituent on the catalytic activity and enantioselectivity are discussed.
\end{abstract}

Keywords: Asymmetric allylic substitution, palladium, phosphites, diamidophosphites, porphyrins.

\section{Применение основанных на гидроксипорфиринах лигандов фосфитного типа к Pd-катализируемым реакциям аллильного замещения}

\author{
К. Н. Гаврилов, ${ }^{a} @$ С. В. Жеглов, ${ }^{a}$ М. Г. Максимова, ${ }^{a}$ И. В. Чучелкин, ${ }^{a}$ \\ И. М. Новиков, ${ }^{a}$ Г. В. Пономарев ${ }^{\mathrm{a}, \mathrm{c}}$ А. Р. Эрзина, ${ }^{\mathrm{a}, \mathrm{b}}$ И. С. Михель ${ }^{\mathrm{a}, \mathrm{b}}$

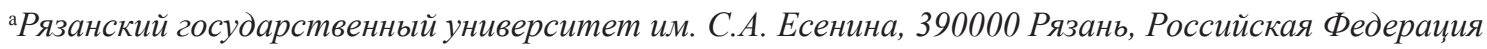 \\ ${ }^{\mathrm{b}}$ Институт физической химии и электрохимии им. А.Н. Фрумкина РАН, 119071 Москва, Российская Федерация \\ 'Научно-исследовательский институт биомедицинской химии им. В.Н. Ореховича, 119121 Москва, Российская \\ Федерация \\ ${ }^{\circledR}$ E-mail: k.gavrilov@rsu.edu.ru
}

\begin{abstract}
На основе (S)-2-(анилинометил)пирролидина или ( $\left.S_{d}\right)$-BINOL полученьл диамидофосфитные и фосфитные лиганды, имеющче порфириновые ичикль. Их использование в Рd-катализируемом асимметрическом алкилировании диметилмалонатом (E)-1,3-дифенилаллилацетата позволило получить до $92 \%$ энантиомерного избытка (ее), в его сульфонилировании пара-толуолсульфинатом натрия - до $82 \%$ ее и в его аминировании пирролидином - до 76 \% ее. Кроме того, до $90 \%$ ее было достигнуто в Рd-катализируемом аллильном алкилировании этил-2-оксоциклогексанкарбоксилатом ицннамилацетата. Обсуждается влияние на каталитическую активность и энантиоселективность таких структурньх особенностей лигандов, как природа фосфорсодержащего ичикла или экзоциклического порфиринового заместителя.
\end{abstract}

Ключевые слова: Асимметрическое аллильное замещение, палладий, фосфиты, диамидофосфиты, порфирины. 


\section{Introduction}

Asymmetric catalysis by transition metal complexes has rapidly grown from the point of a convenient synthetic approach to the organic and organoelement compounds with high enantiomeric purity. Such compounds are widely used as the main components of pharmaceuticals, agrochemicals, perfumery compositions, food additives, and fragrances. ${ }^{[1-6]}$ Activity and enantioselectivity of the metal complex catalysts are determined to a great extent by a proper design and synthesis strategy of the corresponding chiral ligands, first of all, phosphorus containing ligands, thousands representatives of which were used in various asymmetric transformations. ${ }^{[1-}$ ${ }^{13]}$ However, elaboration of an efficient asymmetric inductor for a given catalytic process often represents a challenge. The vast majority of known phosphorus-based chiral ligands in the corresponding metal complexes are able to catalyze (with different enantioselectivities) either a certain type of chemical transformation, or one certain reaction. There are very few versatile (the so-called 'privileged') ligands, and their high cost significantly limits their wide application. Therefore, the development of novel phosphorus-containing stereoselectors is ongoing and still in high demand. ${ }^{[11,14-18]}$

Chiral phosphite-type compounds constitute a class of prominent ligands because they are favorably distinguished by resistance to oxidation, pronounced $\pi$-acidity, and are inexpensive. Also, they can be easily prepared from readily available starting materials via simple condensation processes, including those involving parallel and solidphase synthetic procedures. ${ }^{[5,7,11,12,18-31]}$ In the case of $P^{*}$ chiral phosphite-type ligands the presence of a stereogenic donor of phosphorus atom significantly promotes successful asymmetric induction in the key step of the catalytic cycle. $[2,17,18,32,33]$

Catalytic systems based on chiral supramolecular ligands with phosphite moiety have recently attracted increasing attention. ${ }^{[34-38]}$ In particular, phosphites and phosphoramidites with BINOL or TADDOL backbone and metalloporphyrin fragment have proven to be highly advantageous building blocks in creating large libraries of supramolecular ligands. ${ }^{[34-43]}$ However, according to our best knowledge, there is only one brief mention of chiral phosphite bearing porphyrin core without a metal ion. ${ }^{[40]}$ In this paper, we report on the preparation of a small series of phosphite and diamidophosphite ligands containing metal-free porphyrin framework, that we had subsequently explored in Pd-catalyzed asymmetric allylation reactions.

Pd-catalyzed enantioselective allylic substitutions have become one of the most powerful tools for asymmetric carbon-carbon and carbon-heteroatom bond formation, finding wide application in the total synthesis of valuable enantiomerically pure natural and unnatural compounds. ${ }^{[3,32,44-51]}$ Furthermore, these reactions are the reliable instruments for ligands benchmark testing and the enantiomeric excesses obtained are the simplest scale for evaluating new chiral inductors. ${ }^{[44,50,52]}$ It should also be noted that construction of a quaternary carbon stereocenter via Pdcatalyzed enantioselective synthesis is highly challenging. From a drug discovery perspective, this method provides access to useful building blocks with a single quaternary stereocenter. ${ }^{[53,54]}$

\section{Results and Discussion}

The novel porphyrin-functionalized diamidophosphites 4a-c and phosphite $\mathbf{5}$ were synthesized by one step through the reaction of the corresponding hydroxyporphyrins 1a-c with one equivalent of the phosphorylating reagent $\mathbf{2}$ or $\mathbf{3}$ in the presence of Et ${ }_{3} \mathrm{~N}$ or $i$ - $\mathrm{Pr}_{2} \mathrm{NEt}$ as bases (Scheme 1). Ligands 4a-c and $\mathbf{5}$ were obtained in yields varying from 76 to $85 \%$; they can be stored in the solid form under dry conditions at room temperature for at least a few months with minimal degradation. Compounds $\mathbf{4 a - c}$ and $\mathbf{5}$ were fully characterized by ${ }^{31} \mathrm{P},{ }^{1} \mathrm{H}$, and ${ }^{13} \mathrm{C}$ NMR spectroscopy, MALDI TOF/ TOF mass spectrometry as well as by elemental analysis. Porphyrin-appended ligands $\mathbf{4 a}, \mathbf{4 c}$ and $\mathbf{5}$ are metal-free, diamidophosphite-porphyrinato-zinc(II) compound $\mathbf{4 b}$ was prepared for comparison purposes.

As revealed by ${ }^{31} \mathrm{P}$ and ${ }^{13} \mathrm{C}$ NMR spectroscopy, exclusive formation of stereospecific diamidophosphites 4a-c with $(R)$-configuration at the $P^{*}$-stereocentres occurred (see Table 1 and Experimental). Indeed, the ${ }^{13} \mathrm{C}$ NMR spectra of these ligands are characterized by large spin-spin coupling constants ${ }^{2} J_{\mathrm{C}(8), \mathrm{P}}(35.6-38.5 \mathrm{~Hz})$.

Table 1. ${ }^{31} \mathrm{P}$ NMR chemical shifts and cone angles $\theta$ of ligands 4a-c and 5 .

\begin{tabular}{ccc}
\hline Ligand & $\delta_{\mathrm{p}}, \mathrm{ppm}$ & $\theta$,deg. \\
\hline 4a in $\mathrm{C}_{6} \mathrm{D}_{5} \mathrm{CD}_{3}$ & 123.3 & 121 \\
$\mathbf{4 b}$ in $\mathrm{C}_{6} \mathrm{D}_{5} \mathrm{CD}_{3}$ & 122.8 & 122 \\
$\mathbf{4} \mathbf{c}$ in $\mathrm{C}_{6} \mathrm{D}_{6}$ & 118.3 & 165 \\
$\mathbf{5}$ in $\mathrm{CDCl}_{3}$ & 143.3 & 146 \\
\hline
\end{tabular}

These values suggest the anti-orientation of the pseudoequatorial exocyclic substituent at the phosphorus atom and the $-\left(\mathrm{CH}_{2}\right)_{3}-$ part of the pyrrolidine fragment of the phosphabicyclic skeleton and, consequently, the synorientation of the phosphorus lone pair with respect to the $\mathrm{C}(8)$ atom (Figure 1). ${ }^{[13,18,55-59]}$

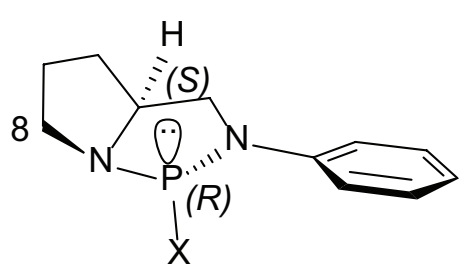

Figure 1. Stereochemistry of the phosphabicyclic part in ligands 4a-c $(\mathrm{X}=$ exocyclic substituent $)$.

In order to have an estimation of the steric bulk of ligands 4a-c and 5, we calculated their Tolman cone angles ${ }^{[60]}$ by the reported method using semi-empirical quantum-mechanical AM1 techniques with full optimization of geometrical parameters. ${ }^{[61]}$ The obtained results (Table 1) show that the steric parameters $(\theta)$ of $\mathbf{4 a - c}$ and $\mathbf{5}$ vary within the interval of $121^{\circ}-165^{\circ}$, peaking at diamidophosphite $4 \mathbf{c}$. Compounds $\mathbf{4 a , b}$ and $\mathbf{5}$ are characterized by moderate steric demands $\left(\theta=121^{\circ}-146^{\circ}\right)$, while $4 \mathbf{c}$ appears to be rather 


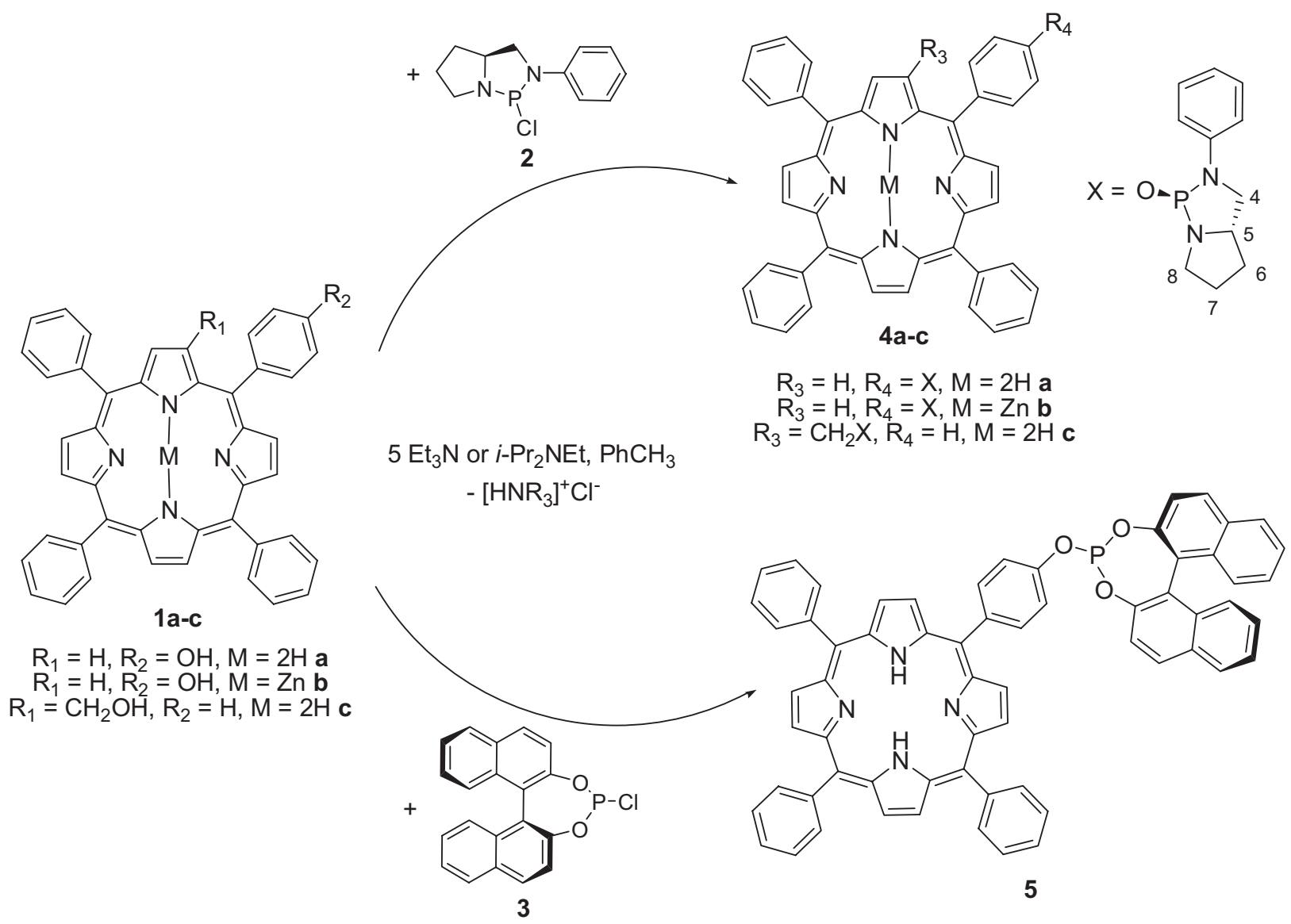

Scheme 1. Synthesis of diamidophosphites $\mathbf{4 a - c}$ and phosphite $\mathbf{5}$.

bulky ligand $\left(\theta=165^{\circ}\right) .^{[60,62,63]}$ It should be noted that ligands bearing $P^{*}$-chirogenic phosphorus atoms and porphyrin or metalloporphyrin fragments were prepared for the first time.

The first set of catalytic runs was carried out employing racemic (E)-1,3-diphenylallyl acetate $\mathbf{6}$, which is a widespread substrate for asymmetric allylic substitutions. As nucleophilic agents, sodium $p$-toluene sulfinate, dimethyl malonate and pyrrolidine were used (Table 2-4). The catalysts were generated in situ from $[\mathrm{Pd}(\mathrm{allyl}) \mathrm{Cl}]_{2}$ and the ligands at $1: 1$ and $2: 1 \mathrm{~L} / \mathrm{Pd}$ molar ratios.

The results achieved with $p$-TolSO $\mathrm{Na}$ as the S-nucleophile are summarized in Table 2. Zinc(II)containing ligand $\mathbf{4 b}$ was the most efficient, and $(S)-\mathbf{7 a}$ was formed with $89 \%$ yield and $82 \%$ ee (Table 2, entry 4$)$. The analogous catalysts based on metal-free diamidophosphite 4a showed a somewhat lower enantioselectivity: up to $70 \%$ ee for $(S)$-7a was achieved in this case (Table 2, entries 1 and 2). Compound $\mathbf{4 c}$ afforded sulfone $(S)-7 \mathbf{a}$ with rather good enantiomeric purity (up to $78 \%$ ee). In general, chemical yield and especially asymmetric induction were poorly sensitive to the $\mathrm{L} / \mathrm{Pd}$ molar ratio. Unfortunately, palladium catalysts with phosphite $\mathbf{5}$ gave no conversion in the synthesis of $7 \mathbf{a}$.

Table 3 shows the results obtained in Pd-catalyzed asymmetric allylic alkylation of substrate $\mathbf{6}$. The most sterically demanding ligand $\mathbf{4 c}$ provided quantitative conversion of the starting substrate and enantioselectivities of up to $92 \%$ (Table 3, entries 9-12). The best result was
Table 2. Pd-catalyzed allylic sulfonylation of (E)-1,3-diphenylallyl acetate $\mathbf{6}$ with sodium $p$-toluene sulfinate ${ }^{\text {a. }}$

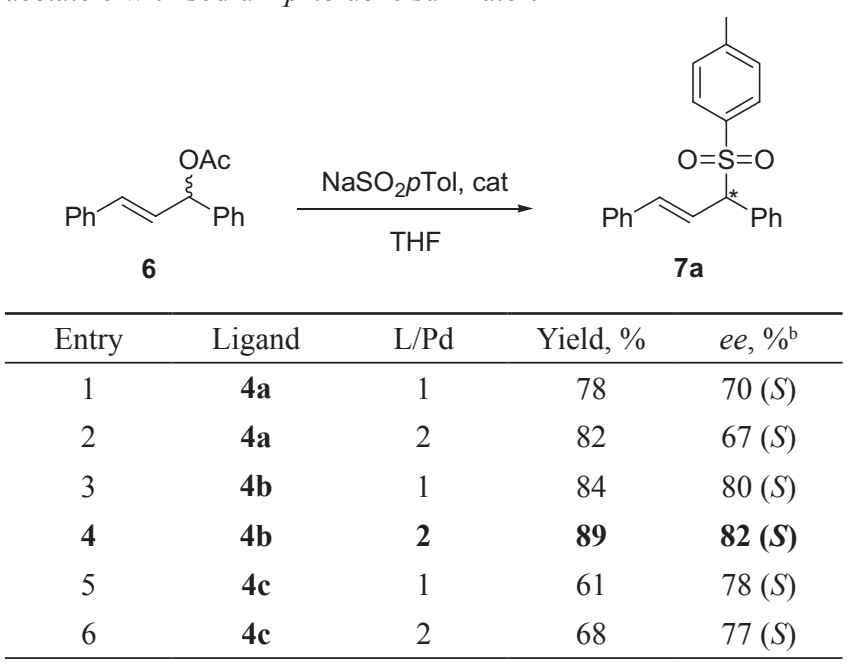

${ }^{\mathrm{a}} \mathrm{All}$ reactions were carried out with $2 \mathrm{~mol} \%$ of $[\mathrm{Pd}(\text { allyl }) \mathrm{Cl}]_{2}$ in THF at room temperature for $48 \mathrm{~h}$.

${ }^{\mathrm{b}}$ Enantiomeric excess of $7 \mathbf{a}$ was determined by HPLC (Daicel Chiralcel OD-H, $\mathrm{C}_{6} \mathrm{H}_{14} / i-\mathrm{PrOH}=4 / 1,0.5 \mathrm{ml} / \mathrm{min}, 254 \mathrm{~nm}, t(R)=$ $16.3 \mathrm{~min}, t(S)=18.5 \mathrm{~min})$.

obtained in $\mathrm{THF}$ at $\mathrm{L} / \mathrm{Pd}=2$. Both diamidophosphites $\mathbf{4 a}$ and $\mathbf{4 b}$ have afforded the desired product $(S)-\mathbf{7 b}$ with $76 \%$ and $75 \%$ ee, respectively. Catalytic systems based on $\mathbf{4 a}$ have demonstrated better activity (Table 3, entries 1-4 and 
5-8). In all cases, with participation of ligands $\mathbf{4 a}$ and $\mathbf{4 b}$, the higher enantioselectivity was observed in $\mathrm{CH}_{2} \mathrm{Cl}_{2}$ as well as at the molar ratio $\mathrm{L} / \mathrm{Pd}=2$. Compound $\mathbf{5}$ has allowed the synthesis of product $(R)-7 \mathbf{b}$ with excellent conversion, but with low enantiomeric purity (no more than $22 \%$ ee).

As a whole, catalytic performance in the Pd-catalyzed allylic amination of $\mathbf{6}$ with pyrrolidine as a N-nucleophile followed the same trend as for its allylic alkylation. In particular, the amination process with participation of diamidophosphite $\mathbf{4} \mathbf{c}$ has resulted in quantitative conversion and enantioselectivity up to $76 \%$ ee (Table 4, entries 9-12). Ligands $\mathbf{4 a}$ and $\mathbf{4 b}$ have provided lower levels of asymmetric induction (64\% and $54 \%$ ee, respectively, Table 4 , entries 3 and 6). Phosphite 5 with binaphthyl backbone was again practically inefficient (ee does not exceed $25 \%$ ).

We have also screened ligands $\mathbf{4 a - c}$ and $\mathbf{5}$ in the allylic alkylation of cinnamyl acetate $\mathbf{8}$ with ethyl 2-oxocyclohexane-1-carboxylate 9 (Table 5); this is a transformation where a quaternary stereogenic center is generated in the nucleophile. The reaction was performed in toluene in the presence of allylpalladium(II) chloride dimer as the precatalyst. Enantioselection occurs when the $\pi$-allyl-ligand complex differentiates between the prochiral faces of the approaching reagent. In this mechanism, where the nucleophile attacks on the face of the $\pi$-allyl system opposite to that of the chirality inducing metal-ligand complex, the generation of asymmetry appears to be relatively challenging. ${ }^{[3,18,53]}$ Both related diamidophosphites $\mathbf{4 a}$ and $\mathbf{4 b}$ have provided practically equal very good results: enantio- meric quaternary-substituted product $(S)$-10 was obtained in $100 \%$ conversion, $88 \%$ ee and $99 \%$ conversion, $90 \%$ ee, respectively (Table 5, entries 2 and 4). Catalysts based on $4 \mathbf{c}$ have proved to be less efficient: $83 \%$ conversion and $78 \%$ ee for $(S)$-10 were achieved in this case. The use of phosphite $\mathbf{5}$ has generated an almost racemic product 10. It is clear that for all ligands the optimal L/Pd molar ratio is 2 .

\section{Conclusion}

In summary, we have designed, synthesized and characterized some novel phosphite-type chiral ligands containing oxyporphyrin groups. In asymmetric Pd-catalyzed allylic substitution, metal-free $P^{*}$-chiral diamidophosphites $\mathbf{4 a}$ and $\mathbf{4 c}$ as well as zinc(II)-containing $\mathbf{4 b}$ are quite efficient as complementary asymmetric inducers. On the contrary, phosphite 5 with $\left(S_{a}\right)$-binaphthyl backbone has provided much lower activity and enantioselectivity. As a whole, diamidophosphite-functionalized porphyrins with stereogenic phosphorus atoms are very attractive for further screening in transition metal catalyzed asymmetric reactions. Such investigations are currently in progress in our laboratories.

\section{Experimental}

${ }^{31} \mathrm{P},{ }^{13} \mathrm{C}$ and ${ }^{1} \mathrm{H}$ NMR spectra were recorded on a Bruker Avance III $600\left(242.9 \mathrm{MHz}\right.$ for ${ }^{31} \mathrm{P}, 150.9 \mathrm{MHz}$ for ${ }^{13} \mathrm{C}$ and $600.13 \mathrm{MHz}$

Table 3. Pd-catalyzed allylic alkylation of $(E)$-1,3-diphenylallyl acetate 6 with dimethyl malonate .

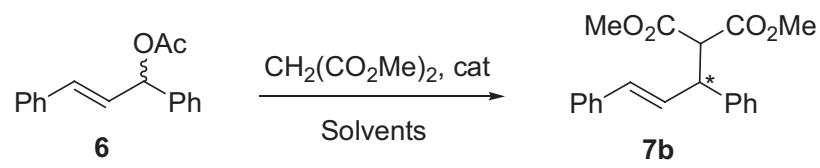

\begin{tabular}{|c|c|c|c|c|c|}
\hline Entry & Ligand & $\mathrm{L} / \mathrm{Pd}$ & Solvent & Conversion, $\%$ & $e e, \%^{b}$ \\
\hline 1 & $4 a$ & 1 & $\mathrm{CH}_{2} \mathrm{Cl}_{2}$ & 100 & $69(S)$ \\
\hline 2 & $4 a$ & 2 & $\mathrm{CH}_{2} \mathrm{Cl}_{2}$ & 100 & $76(S)$ \\
\hline 3 & $4 a$ & 1 & THF & 91 & $67(S)$ \\
\hline 4 & $4 a$ & 2 & THF & 90 & $69(S)$ \\
\hline 5 & $4 b$ & 1 & $\mathrm{CH}_{2} \mathrm{Cl}_{2}$ & 88 & $68(S)$ \\
\hline 6 & $4 b$ & 2 & $\mathrm{CH}_{2} \mathrm{Cl}_{2}$ & 62 & $75(S)$ \\
\hline 7 & $4 b$ & 1 & THF & 87 & $62(S)$ \\
\hline 8 & $4 b$ & 2 & THF & 53 & $70(S)$ \\
\hline 9 & $4 c$ & 1 & $\mathrm{CH}_{2} \mathrm{Cl}_{2}$ & 100 & $83(S)$ \\
\hline 10 & $4 c$ & 2 & $\mathrm{CH}_{2} \mathrm{Cl}_{2}$ & 100 & $84(S)$ \\
\hline 11 & $4 c$ & 1 & THF & 100 & $87(S)$ \\
\hline 12 & $4 c$ & 2 & THF & 100 & $92(S)$ \\
\hline 13 & 5 & 1 & $\mathrm{CH}_{2} \mathrm{Cl}_{2}$ & 98 & $17(R)$ \\
\hline 14 & 5 & 2 & $\mathrm{CH}_{2} \mathrm{Cl}_{2}$ & 94 & $22(R)$ \\
\hline 15 & 5 & 1 & THF & 0 & - \\
\hline 16 & 5 & 2 & THF & 0 & - \\
\hline
\end{tabular}

${ }^{\mathrm{a}} \mathrm{All}$ reactions were carried out with $2 \mathrm{~mol} \%$ of $[\mathrm{Pd}(\mathrm{allyl}) \mathrm{Cl}]_{2}$ at room temperature for $48 \mathrm{~h}$ (BSA, KOAc).

${ }^{b}$ The conversion of substrate 6 and enantiomeric excess of $7 \mathbf{b}$ were determined by HPLC (Daicel Chiralcel OD-H, $\mathrm{C}_{6} \mathrm{H}_{14} / i-\mathrm{PrOH}=99 / 1$, $0.3 \mathrm{ml} / \mathrm{min}, 254 \mathrm{~nm}, t(R)=28.0 \mathrm{~min}, t(S)=29.3 \mathrm{~min})$. 
Hydroxyporphyrins-based Ligands at Asymmetric Substitution Reactions

Table 4. Pd-catalyzed allylic amination of (E)-1,3-diphenylallyl acetate 6 with pyrrolidine ${ }^{\text {a }}$<smiles>CC(=O)O[C@H](/C=C/c1ccccc1)c1ccccc1</smiles>

6

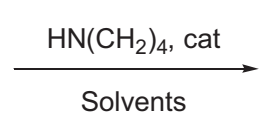

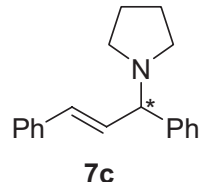

\begin{tabular}{|c|c|c|c|c|c|}
\hline Entry & Ligand & $\mathrm{L} / \mathrm{Pd}$ & Solvent & Conversion, $\%$ & $e e, \%^{\mathrm{b}}$ \\
\hline 1 & $4 a$ & 1 & $\mathrm{CH}_{2} \mathrm{Cl}_{2}$ & 100 & $53(R)$ \\
\hline 2 & $4 a$ & 2 & $\mathrm{CH}_{2} \mathrm{Cl}_{2}$ & 100 & $54(R)$ \\
\hline 3 & $4 a$ & 1 & THF & 80 & $64(R)$ \\
\hline 4 & $4 a$ & 2 & THF & 75 & $55(R)$ \\
\hline 5 & $4 b$ & 1 & $\mathrm{CH}_{2} \mathrm{Cl}_{2}$ & 99 & $44(R)$ \\
\hline 6 & $4 b$ & 2 & $\mathrm{CH}_{2} \mathrm{Cl}_{2}$ & 96 & $54(R)$ \\
\hline 7 & $4 b$ & 1 & THF & 71 & $48(R)$ \\
\hline 8 & $4 b$ & 2 & THF & 89 & $52(R)$ \\
\hline 9 & $4 c$ & 1 & $\mathrm{CH}_{2} \mathrm{Cl}_{2}$ & 100 & $70(R)$ \\
\hline 10 & $4 c$ & 2 & $\mathrm{CH}_{2} \mathrm{Cl}_{2}$ & 100 & $75(R)$ \\
\hline 11 & $4 c$ & 1 & THF & 100 & $75(R)$ \\
\hline 12 & $4 c$ & 2 & THF & 100 & $76(R)$ \\
\hline 13 & 5 & 1 & $\mathrm{CH}_{2} \mathrm{Cl}_{2}$ & 24 & $10(R)$ \\
\hline 14 & 5 & 2 & $\mathrm{CH}_{2} \mathrm{Cl}_{2}$ & 30 & $5(R)$ \\
\hline 15 & 5 & 1 & THF & 10 & $25(R)$ \\
\hline 16 & 5 & 2 & THF & 19 & $16(R)$ \\
\hline
\end{tabular}

${ }^{a} \mathrm{All}$ reactions were carried out with $2 \mathrm{~mol} \%$ of $[\mathrm{Pd}(\text { allyl }) \mathrm{Cl}]_{2}$ at room temperature for $48 \mathrm{~h}$.

${ }^{b}$ The conversion of substrate 6 and enantiomeric excess of $7 \mathbf{c}$ were determined by HPLC (Daicel Chiralcel OD- $\mathrm{H}_{2} \mathrm{C}_{6} \mathrm{H}_{14} / i-\mathrm{PrOH} / \mathrm{HN}(\mathrm{Et})_{2}=$ $200 / 1 / 0.1,0.9 \mathrm{ml} / \mathrm{min}, 254 \mathrm{~nm}, t(R)=5.0 \mathrm{~min}, t(S)=6.1 \mathrm{~min})$.

Table 5. Pd-catalyzed allylic alkylation of cinnamyl acetate $\mathbf{8}$ with ethyl 2-oxocyclohexane-1-carboxylate $\mathbf{9}^{\text {a }}$.

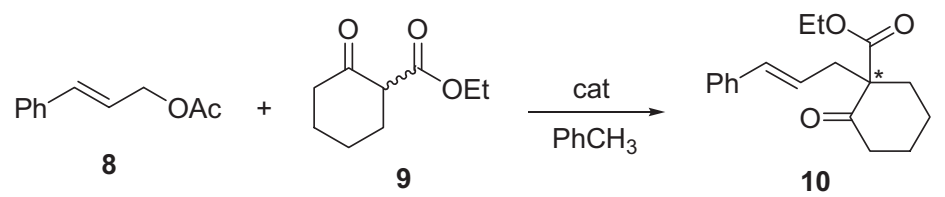

\begin{tabular}{|c|c|c|c|c|}
\hline Entry & Ligand & $\mathrm{L} / \mathrm{Pd}$ & Conversion, $\%$ & $e e, \%^{\mathrm{b}}$ \\
\hline 1 & $4 a$ & 1 & 68 & $74(S)$ \\
\hline 2 & $4 a$ & 2 & 100 & $88(S)$ \\
\hline 3 & $4 b$ & 1 & 55 & $68(S)$ \\
\hline 4 & $4 b$ & 2 & 99 & $90(S)$ \\
\hline 5 & $4 c$ & 1 & 58 & $55(S)$ \\
\hline 6 & $4 c$ & 2 & 83 & $78(S)$ \\
\hline 7 & 5 & 1 & 0 & - \\
\hline 8 & 5 & 2 & 16 & $12(S)$ \\
\hline
\end{tabular}

${ }^{a} A l l$ reactions were carried out with $2 \mathrm{~mol} \%$ of $[\mathrm{Pd}(\text { allyl }) \mathrm{Cl}]_{2}$ in toluene at room temperature for $48 \mathrm{~h}\left(\mathrm{BSA}, \mathrm{Zn}(\mathrm{OAc})_{2}\right)$.

${ }^{b}$ The conversion of substrate $\mathbf{8}$ and enantiomeric excess of $\mathbf{1 0}$ were determined by HPLC (Kromasil 5-CelluCoat, $\mathrm{C}_{6} \mathrm{H}_{14} / i-\mathrm{PrOH}_{2}=95 / 5,0.4$ $\mathrm{ml} / \mathrm{min}, 254 \mathrm{~nm}, t(R)=14.3 \mathrm{~min}, t(S)=16.4 \mathrm{~min})$. 
for ${ }^{1} \mathrm{H}$ ) spectrometer. The assignment of the resonances in the ${ }^{1} \mathrm{H}$ and ${ }^{13} \mathrm{C}$ NMR spectra was achieved by the use of DEPT, COSY, and HSQC techniques and published data. ${ }^{[55-57]}$ Chemical shifts $(\mathrm{ppm})$ are given relative to $\mathrm{Me}_{4} \mathrm{Si}\left({ }^{1} \mathrm{H}\right.$ and $\left.{ }^{13} \mathrm{C}\right)$ and $85 \% \mathrm{H}_{3} \mathrm{PO}_{4}\left({ }^{31} \mathrm{P}\right.$ NMR). Data are represented as follows: chemical shift, multiplicity ( $\mathrm{br}=$ broad, $\mathrm{s}=$ singlet, $\mathrm{d}=$ doublet, $\mathrm{t}=$ triplet, $\mathrm{m}=$ multiplet); coupling constants ${ }^{\mathrm{n}} J$ in $\mathrm{Hz}$ integration, «n» values are reported in the case of their unambiguous determination. Mass spectra were recorded on a Bruker Daltonics Ultraflex spectrometer (MALDI TOF/TOF). Optical rotations were measured on an Atago AP300 polarimeter. HPLC analyses were performed on Agilent 1100 and Stayer instruments using Chiralcel ${ }^{\circledR}$ and Kromasil ${ }^{\circledR}$ columns. Elemental analyses were performed on a $\mathrm{CHN}$-microanalyzer Carlo Erba EA1108 CHNS-O.

All reactions were carried out under a dry argon atmosphere in flame-dried glassware and in freshly dried and distilled solvents. For example, toluene and tetrahydrofuran were freshly distilled from sodium benzophenone ketyl before use; dichloromethane was distilled from $\mathrm{NaH}$. Triethylamine, $\mathrm{N}, \mathrm{N}$ diisopropylethylamine and pyrrolidine were distilled over $\mathrm{KOH}$ and then over a small amount of $\mathrm{LiAlH}_{4}$ before use. Thin-layer chromatography was performed on E. Merck pre-coated silica gel 60 F254 and Macherey-Nagel Alugram Alox N/UV ${ }_{254}$ plates. Column chromatography was performed using silica gel MN Kieselgel 60 (230-400 mesh) and MN-Aluminum oxide, basic, Brockmann Activity I. 4-(10,15,20-Triphenylporphyrin-5-yl) phenol 1a, 5-(4-hydroxyphenyl)-10,15,20-triphenylporphyrinyl$\kappa^{4} N^{21}, N^{22}, N^{23}, N^{24}$-zinc(II) 1 b and (5,10,15,20-tetraphenylporphyrin2-yl)methanol 1c were prepared using known procedures. ${ }^{[42,64-66]}$ Phosphorylating reagents (5S)-2-chloro-3-phenyl-1,3-diaza-2phosphabicyclo[3.3.0]octane 2 and $\left(S_{a}\right)$-4-chlorodinaphtho[2,1$\left.d: 1^{\prime}, 2^{\prime}-f\right][1,3,2]$ dioxaphosphepine $\mathbf{3}$ were prepared as published. $[56,67]$ The $[\mathrm{Pd}(\text { allyl }) \mathrm{Cl}]_{2}$ and starting substrate $\mathbf{6}$ were obtained as published. ${ }^{\left[{ }^{[8]}\right.} \mathrm{Pd}$-catalyzed reactions: allylic sulfonylation of $(E)-1,3-$ diphenylallyl acetate $\mathbf{6}$ with sodium $p$-toluene sulfinate, alkylation with dimethyl malonate, amination with pyrrolidine and allylic alkylation of cinnamyl acetate $\mathbf{8}$ with ethyl 2-oxocyclohexane1-carboxylate 9 were performed according to the appropriate procedures. ${ }^{[53,56,69]}$

$\left(S_{a}\right)$-BINOL, sodium $p$-toluene sulfinate, dimethyl malonate, BSA ( $N, O$-bis(trimethylsilyl) acetamide), cinnamyl acetate 8 and ethyl 2-oxocyclohexane-1-carboxylate 9 were purchased from Aldrich and Acros Organics and used without further purification.

General procedure for the preparation of ligands $\mathbf{4 a - c}$ and $\mathbf{5}$. To a vigorously stirred solution of the appropriate phosphorylating reagent $(1 \mathrm{mmol})$ and $\mathrm{Et}_{3} \mathrm{~N}(0.70 \mathrm{ml}, 5 \mathrm{mmol})$ or $i$-Pr $\mathrm{Pr}_{2} \mathrm{NEt}$ (in the synthesis of $4 \mathbf{b}, 0.87 \mathrm{ml}, 5 \mathrm{mmol})$ in toluene $(15 \mathrm{ml})$ relevant hydroxyporphyrin $(1 \mathrm{mmol})$ was added in one portion. The mixture was stirred at reflux for $5 \mathrm{~min}$, and cooled to $20^{\circ} \mathrm{C}$. The resulting suspension was filtered through a short plug of aluminum oxide, the column was washed twice with toluene $(5 \mathrm{ml})$, and the solvent was evaporated under reduced pressure (40 Torr). The residue was additionally purified by flash chromatography on silica gel (toluene)

(2R,5S)-2-(4-(10,15,20-Triphenylporphyrin-5-yl)phenoxy)-3phenyl-1,3-diaza-2-phosphabicyclo [3.3.0]octane (4a). Maroonpurple powder $(0.69 \mathrm{~g}$, yield $83 \%)$. [a $]_{\mathrm{D}}^{23}=-214.5^{\circ}(c 1.0$, toluene). Found, \%: C, 79.46; H, 5.42; N, 10.17. $\mathrm{C}_{55} \mathrm{H}_{43} \mathrm{~N}_{6} \mathrm{OP}$. Calculated, \%: C, 79.12; H, 5.19; N, 10.07. Mass spectra (MALDI TOF/TOF) $m / z(\%): 835(100)\left([\mathrm{M}+\mathrm{H}]^{+}\right), 631(52)\left(\left[\mathrm{C}_{44} \mathrm{H}_{30} \mathrm{~N}_{4} \mathrm{O}+\mathrm{H}\right]^{+}\right) .{ }^{1} \mathrm{H}$ NMR (600.13 MHz, $\left.\mathrm{C}_{6} \mathrm{D}_{5} \mathrm{CD}_{3}, 25{ }^{\circ} \mathrm{C}\right) \delta_{\mathrm{H}}$ ppm: -2.29 (br. s, $2 \mathrm{H}$, $\mathrm{NH}), 1.11-1.17\left(\mathrm{~m}, 1 \mathrm{H}, \mathrm{C}(6) \mathrm{H}_{2}\right), 1.40-1.48\left(\mathrm{~m}, 2 \mathrm{H}, \mathrm{C}(7) \mathrm{H}_{2}\right)$, 1.56-1.61 (m, $\left.1 \mathrm{H}, \mathrm{C}(6) \mathrm{H}_{2}\right), 2.88-2.92\left(\mathrm{~m}, 1 \mathrm{H}, \mathrm{C}(4) \mathrm{H}_{2}\right), 3.10-3.16$ $\left(\mathrm{m}, 1 \mathrm{H}, \mathrm{C}(8) \mathrm{H}_{2}\right), 3.42\left(\mathrm{dd},{ }^{3} J_{\mathrm{H}, \mathrm{H}}=7.8,{ }^{3} J_{\mathrm{H}, \mathrm{H}}=9.0,1 \mathrm{H}, \mathrm{C}(4) \mathrm{H}_{2}\right)$, $3.46-3.52\left(\mathrm{~m}, 1 \mathrm{H}, \mathrm{C}(8) \mathrm{H}_{2}\right), 3.76-3.81(\mathrm{~m}, 1 \mathrm{H}, \mathrm{C}(5) \mathrm{H}), 6.89$ (tt, ${ }^{3} J_{\mathrm{H}, \mathrm{H}}=6.6, J_{\mathrm{H}, \mathrm{H}}=1.8,1 \mathrm{H}, \mathrm{Ar}$ ) $, 7.26-7.31(\mathrm{~m}, 4 \mathrm{H}, \mathrm{Ar}),. 7.34(\mathrm{~d}$, $\left.{ }^{3} J_{\mathrm{H}, \mathrm{H}}=8.4,2 \mathrm{H}, \mathrm{Ar}.\right), 7.44-7.50$ (m, $9 \mathrm{H}, \mathrm{Ar}$ ), $7.93\left(\mathrm{~d},{ }^{3} J_{\mathrm{H}, \mathrm{H}}=8.4\right.$, 2 H, Ar.), 8.07-8.11 (m, 6 H, Ar.), 8.85 (br. s, 4 H, Ar.), 8.88 (d, $\left.{ }^{3} J_{\mathrm{H}, \mathrm{H}}=4.8,2 \mathrm{H}, \mathrm{Ar}.\right), 8.90$ (d, ${ }^{3} J_{\mathrm{H}, \mathrm{H}}=4.8,2 \mathrm{H}, \mathrm{Ar}$ ). ${ }^{13} \mathrm{C} \mathrm{NMR}(150.9$ $\left.\mathrm{MHz}, \mathrm{C}_{6} \mathrm{D}_{5} \mathrm{CD}_{3}, 25{ }^{\circ} \mathrm{C}\right) \delta_{\mathrm{C}} \mathrm{ppm:} 26.6\left(\mathrm{~d},{ }^{3} J_{\mathrm{CP}}=4.5, \mathrm{C}(7)\right), 32.1(\mathrm{~s}$, $\mathrm{C}(6)), 48.0\left(\mathrm{~d},{ }^{2} J_{\mathrm{C}, \mathrm{P}}=35.6, \mathrm{C}(8)\right), 54.3\left(\mathrm{~d},{ }^{2} J_{\mathrm{C}, \mathrm{P}}=8.1, \mathrm{C}(4)\right), 63.5(\mathrm{~d}$, $\left.\left.{ }^{2} J_{\mathrm{C}, \mathrm{P}}=8.8, \mathrm{C}(5)\right), 115.9\left(\mathrm{~d},{ }^{3} J_{\mathrm{C}, \mathrm{P}}=13.0, \mathrm{CH}_{\mathrm{Ar}}\right)^{\mathrm{C}}\right), 119.9\left(\mathrm{~d}, J_{\mathrm{C}, \mathrm{P}}=0.9\right.$, $\left.\mathrm{CH}_{\mathrm{Ar}}\right), 120.46\left(\mathrm{~s}, \mathrm{C}_{\mathrm{Ar}}\right), 120.52\left(\mathrm{~s}, \mathrm{C}_{\mathrm{Ar}}\right), 120.7\left(\mathrm{~d}, J_{\mathrm{C}, \mathrm{P}}=4.7, \mathrm{CH}_{\mathrm{Ar}}\right)$, $126.9\left(\mathrm{~s}, \mathrm{CH}_{\mathrm{Ar}}\right), 127.8\left(\mathrm{~s}, \mathrm{CH}_{\mathrm{Ar}}\right), 129.7\left(\mathrm{~s}, \mathrm{CH}_{\mathrm{Ar}}\right), 131.4$ (very br., $\left.\mathrm{CH}_{\mathrm{Ar}}\right), 134.9\left(\mathrm{~s}, \mathrm{CH}_{\mathrm{Ar}}\right), 135.7\left(\mathrm{~s}, \mathrm{CH}_{\mathrm{Ar}}\right), 137.6\left(\mathrm{~s}, \mathrm{C}_{\mathrm{Ar}}\right), 142.96(\mathrm{~s}$ $\left.\mathrm{C}_{\mathrm{Ar}}\right), 143.0\left(\mathrm{~s}, \mathrm{C}_{\mathrm{Ar}}\right), 146.0\left(\mathrm{~d},{ }^{2} J_{\mathrm{C}, \mathrm{P}}=15.2, \mathrm{C}_{\mathrm{Ph}}\right), 154.6\left(\mathrm{~d},{ }^{2} J_{\mathrm{C}, \mathrm{P}}=5.3\right.$, POC). ${ }^{31} \mathrm{P}$ NMR (242.9 MHz, $\left.\mathrm{C}_{6} \mathrm{D}_{5} \mathrm{CD}_{3}, 25{ }^{\circ} \mathrm{C}\right) \delta_{\mathrm{p}} \mathrm{ppm}$ : 123.3 .

$(2 R, 5 S)-2-(4-(10,15,20-$ Triphenyl-zinc(II) porphyrin-5-yl) phenoxy)-3-phenyl-1,3-diaza-2-phosphabicyclo[3.3.0]octane (4b). Maroon-purple powder $(0.68 \mathrm{~g}$, yield $76 \%) .[\mathrm{a}]_{\mathrm{D}}^{23}=-262.2^{\circ}(c$ 1.0 , toluene). Found, \%: C, 73.83; H, 4.74; N, 9.04. $\mathrm{C}_{55} \mathrm{H}_{41} \mathrm{~N}_{6} \mathrm{OPZn}$. Calculated, \%: C, 73.54; H, 4.60; N, 9.36. Mass spectra (MALDI TOF/TOF) $m / z(\%): 897(10)\left([\mathrm{M}+\mathrm{H}]^{+}\right), 693(12)\left(\left[\mathrm{C}_{44} \mathrm{H}_{28} \mathrm{~N}_{4} \mathrm{OZn}\right.\right.$ $\left.+\mathrm{H}]^{+}\right), 206(100)\left(\left[\mathrm{C}_{11} \mathrm{H}_{15} \mathrm{~N}_{2} \mathrm{P}\right]^{+}\right) .{ }^{1} \mathrm{H} \mathrm{NMR}\left(600.13 \mathrm{MHz}, \mathrm{C}_{6} \mathrm{D}_{5} \mathrm{CD}_{3}\right.$, $\left.23{ }^{\circ} \mathrm{C}\right) \delta_{\mathrm{H}} \mathrm{ppm}: 1.16-1.22\left(\mathrm{~m}, 1 \mathrm{H}, \mathrm{C}(6) \mathrm{H}_{2}\right), 1.46-1.54(\mathrm{~m}, 2 \mathrm{H}$, $\left.\mathrm{C}(7) \mathrm{H}_{2}\right), 1.62-1.68\left(\mathrm{~m}, 1 \mathrm{H}, \mathrm{C}(6) \mathrm{H}_{2}\right), 2.93-2.97$ (m, $1 \mathrm{H}, \mathrm{C}(4)$ $\left.\mathrm{H}_{2}\right), 3.16-3.21\left(\mathrm{~m}, 1 \mathrm{H}, \mathrm{C}(8) \mathrm{H}_{2}\right), 3.47\left(\mathrm{dd},{ }^{3} J_{\mathrm{H}, \mathrm{H}}=7.8,{ }^{3} J_{\mathrm{H}, \mathrm{H}}=9.0\right.$, $\left.1 \mathrm{H}, \mathrm{C}(4) \mathrm{H}_{2}\right), 3.50-3.56\left(\mathrm{~m}, 1 \mathrm{H}, \mathrm{C}(8) \mathrm{H}_{2}\right), 3.81-3.86(\mathrm{~m}, 1 \mathrm{H}, \mathrm{C}(5)$ $\mathrm{H}), 6.94\left(\mathrm{tt},{ }^{3} J_{\mathrm{H}, \mathrm{H}}=6.6, J_{\mathrm{H}, \mathrm{H}}=1.8,1 \mathrm{H}, \mathrm{Ar}\right), 7.30-7.33(\mathrm{~m}, 4 \mathrm{H}$, Ar.), 7.35 (d, ${ }^{3} J_{\mathrm{H}, \mathrm{H}}=8.4,2 \mathrm{H}, \mathrm{Ar}$ ), 7.67-7.61 (m, $9 \mathrm{H}, \mathrm{Ar}$ ), 8.10 (d, ${ }^{3} J_{\mathrm{H}, \mathrm{H}}=8.4,2 \mathrm{H}, \mathrm{Ar}$ ), $8.28-8.31$ (m, $6 \mathrm{H}, \mathrm{Ar}$ ), 9.04 (br. s, $4 \mathrm{H}$, Ar.), 9.07 (d, ${ }^{3} J_{\mathrm{H}, \mathrm{H}}=4.8,2 \mathrm{H}, \mathrm{Ar}$.), 9.08 (d, ${ }^{3} J_{\mathrm{H}, \mathrm{H}}=4.8,2 \mathrm{H}, \mathrm{Ar}$.). ${ }^{13} \mathrm{C}$ NMR (150.9 MHz, $\left.\mathrm{C}_{6} \mathrm{D}_{5} \mathrm{CD}_{3}, 23{ }^{\circ} \mathrm{C}\right) \delta_{\mathrm{C}} \mathrm{ppm}: 26.6\left(\mathrm{~d},{ }^{3} J_{\mathrm{C}, \mathrm{P}}=4.4\right.$ $\mathrm{C}(7)), 32.2(\mathrm{~s}, \mathrm{C}(6)), 48.0\left(\mathrm{~d},{ }^{2} J_{\mathrm{CP}}=35.7, \mathrm{C}(8)\right), 54.4\left(\mathrm{~d},{ }^{2} J_{\mathrm{CP}}=8.1\right.$, $\mathrm{C}(4)), 63.5\left(\mathrm{~d},{ }^{2} J_{\mathrm{C}, \mathrm{P}}=8.9, \mathrm{C}(5)\right), 115.9\left(\mathrm{~d},{ }^{3} J_{\mathrm{C}, \mathrm{P}}=13.0, \mathrm{CH}_{\mathrm{Ar}}\right), 119.8$ $\left(\mathrm{s}, \mathrm{CH}_{\mathrm{Ar}}\right), 120.6\left(\mathrm{~d}, J_{\mathrm{C}, \mathrm{P}}=4.2, \mathrm{CH}_{\mathrm{Ar}}\right), 120.97\left(\mathrm{~s}, \mathrm{C}_{\mathrm{Ar}}\right), 121.0\left(\mathrm{~s}, \mathrm{C}_{\mathrm{Ar}}\right)$, $121.1\left(\mathrm{~s}, \mathrm{C}_{\mathrm{Ar}}\right), 126.7\left(\mathrm{~s}, \mathrm{CH}_{\mathrm{Ar}}\right), 127.5\left(\mathrm{~s}, \mathrm{CH}_{\mathrm{Ar}}\right), 129.7\left(\mathrm{~s}, \mathrm{CH}_{\mathrm{Ar}}\right)$, $132.0\left(\mathrm{~s}, \mathrm{CH}_{\mathrm{Ar}}\right), 132.1\left(\mathrm{~s}, \mathrm{CH}_{\mathrm{Ar}}\right), 134.9\left(\mathrm{~s}, \mathrm{CH}_{\mathrm{Ar}}\right), 135.6\left(\mathrm{~s}, \mathrm{CH}_{\mathrm{Ar}}\right)$, $137.6\left(\mathrm{~s}, \mathrm{C}_{\mathrm{Ar}}\right), 143.99\left(\mathrm{~s}, \mathrm{C}_{\mathrm{Ar}}\right), 144.0\left(\mathrm{~s}, \mathrm{C}_{\mathrm{Ar}}\right), 150.45\left(\mathrm{~d},{ }^{2} J_{\mathrm{CP}}=2.3\right.$, POC), $150.6\left(\mathrm{~d},{ }^{2} J_{\mathrm{C}, \mathrm{P}}=15.8, \mathrm{C}_{\mathrm{Ph}}\right) .{ }^{31} \mathrm{P} \mathrm{NMR}\left(242.9 \mathrm{MHz}, \mathrm{C}_{6} \mathrm{D}_{5} \mathrm{CD}_{3}\right.$, $\left.23{ }^{\circ} \mathrm{C}\right) \delta_{\mathrm{p}} \mathrm{ppm}: 122.8$.

(2R,5S)-2-((5,10,15,20-Tetraphenylporphyrin-2-yl)methoxy)3-phenyl-1,3-diaza-2-phosphabicyclo [3.3.0]octane (4c). Maroonpurple powder $(0.72 \mathrm{~g}$, yield $85 \%)$. [a $]_{\mathrm{D}}{ }^{23}=-193.2^{\circ}$ (c 1.0, toluene). Found, \%: C, 79.52; H, 5.44; N, 10.07. $\mathrm{C}_{56} \mathrm{H}_{45} \mathrm{~N}_{6} \mathrm{OP}$. Calculated, \%: C, 79.23; H, 5.34; N, 9.90. Mass spectra (MALDI TOF/TOF) $\mathrm{m} / \mathrm{z}$ (\%): $849(11)\left([\mathrm{M}+\mathrm{H}]^{+}\right), 629(100)\left(\left[\mathrm{C}_{45} \mathrm{H}_{32} \mathrm{~N}_{4}+\mathrm{H}\right]^{+}\right) .{ }^{1} \mathrm{H}$ NMR $\left(600.13 \mathrm{MHz}, \mathrm{C}_{6} \mathrm{D}_{6}, 27^{\circ} \mathrm{C}\right) \delta_{\mathrm{H}}$ ppm: -2.15 (br. s, $\left.2 \mathrm{H}, \mathrm{NH}\right), 1.13-$ $1.18\left(\mathrm{~m}, 1 \mathrm{H}, \mathrm{C}(6) \mathrm{H}_{2}\right), 1.27-1.39\left(\mathrm{~m}, 2 \mathrm{H}, \mathrm{C}(7) \mathrm{H}_{2}\right), 1.55-2.61(\mathrm{~m}$, $\left.1 \mathrm{H}, \mathrm{C}(6) \mathrm{H}_{2}\right), 2.83-2.90\left(\mathrm{~m}, 2 \mathrm{H}, \mathrm{C}(4) \mathrm{H}_{2}\right.$ and $\left.\mathrm{C}(8) \mathrm{H}_{2}\right), 3.33-3.39$ $\left(\mathrm{m}, 2 \mathrm{H}, \mathrm{C}(4) \mathrm{H}_{2}\right.$ and $\left.\mathrm{C}(8) \mathrm{H}_{2}\right), 3.66-3.71(\mathrm{~m}, 1 \mathrm{H}, \mathrm{C}(5) \mathrm{H}), 4.97$ $\left(\mathrm{dd},{ }^{2} J_{\mathrm{H}, \mathrm{H}}=15.0,{ }^{3} J_{\mathrm{H} . \mathrm{P}}=5.4,1 \mathrm{H}, \mathrm{CH}_{2} \mathrm{O}\right), 5.15\left(\mathrm{dd},{ }^{2} J_{\mathrm{H} . \mathrm{H}}=15.0\right.$, $\left.{ }^{3} J_{\mathrm{H}, \mathrm{P}}=6.0,1 \mathrm{H}, \mathrm{CH}_{2} \mathrm{O}\right), 6.81\left(\mathrm{t},{ }^{3} J_{\mathrm{H}, \mathrm{H}}=6.6,1 \mathrm{H}, \mathrm{CH}_{\mathrm{Ph}}\right), 7.16-7.20$ $\left(\mathrm{m}, 4 \mathrm{H}, \mathrm{CH}_{\mathrm{Ph}}\right), 7.27\left(\mathrm{t},{ }^{3} J_{\mathrm{H}, \mathrm{H}}=7.2,1 \mathrm{H}, \mathrm{CH}_{\mathrm{Ph}}\right), 7.36\left(\mathrm{t},{ }^{3} J_{\mathrm{H}, \mathrm{H}}=7.8\right.$, $\left.1 \mathrm{H}, \mathrm{CH}_{\mathrm{ph}}\right), 7.39-7.48\left(\mathrm{~m}, 10 \mathrm{H}, \mathrm{CH}_{\mathrm{ph}}\right), 7.55\left(\mathrm{~d},{ }^{3} J_{\mathrm{H}, \mathrm{H}}=7.2,1 \mathrm{H}\right.$, $\left.\mathrm{CH}_{\mathrm{Ph}}\right), 7.74\left(\mathrm{~d},{ }^{3} J_{\mathrm{H} . \mathrm{H}}=7.8,1 \mathrm{H}, \mathrm{CH}_{\mathrm{Ph}}\right), 8.08-8.12\left(\mathrm{~m}, 4 \mathrm{H}, \mathrm{CH}_{\mathrm{Ph}}\right)$, $8.16\left(\mathrm{~d},{ }^{3} \mathrm{~J}_{\mathrm{H}, \mathrm{H}}=7.2,2 \mathrm{H}, \mathrm{CH}_{\mathrm{Ph}}\right), 8.62\left(\mathrm{~d},{ }^{3} \mathrm{~J}_{\mathrm{H}, \mathrm{H}}=4.8,1 \mathrm{H}, \mathrm{Ar}.\right)$, $8.86\left(\mathrm{~d},{ }^{3} J_{\mathrm{H}, \mathrm{H}}=4.8,1 \mathrm{H}, \mathrm{Ar}.\right), 8.89\left(\mathrm{~d},{ }^{3} J_{\mathrm{H}, \mathrm{H}}=4.8,1 \mathrm{H}, \mathrm{Ar}.\right), 8.90$ (d, ${ }^{3} J_{\mathrm{H}, \mathrm{H}}=4.8,1 \mathrm{H}$, Ar.), $8.904\left(\mathrm{~d},{ }^{3} J_{\mathrm{H}, \mathrm{H}}=5.4,1 \mathrm{H}, \mathrm{Ar}\right.$ ), 8.913 (d, $\left.{ }^{3} J_{\mathrm{H}, \mathrm{H}}=5.4,1 \mathrm{H}, \mathrm{Ar}.\right), 9.56\left(\mathrm{~s}, 1 \mathrm{H}, \mathrm{OCH}_{2} \mathrm{CCH}\right) .{ }^{13} \mathrm{C} \mathrm{NMR}(150.9$ $\left.\mathrm{MHz}, \mathrm{C}_{6} \mathrm{D}_{6}, 27^{\circ} \mathrm{C}\right) \delta_{\mathrm{C}} \mathrm{ppm}: 26.0\left(\mathrm{~d}, 3^{2} J_{\mathrm{CP}}=3.8, \mathrm{C}(7)\right), 31.8(\mathrm{~s}$, $\mathrm{C}(6)), 48.3\left(\mathrm{~d},{ }^{2} J_{\mathrm{C}, \mathrm{P}}=38.5, \mathrm{C}(8)\right), 54.9\left(\mathrm{~d},{ }^{2} J_{\mathrm{C}, \mathrm{P}}=7.5, \mathrm{C}(4)\right), 60.7(\mathrm{~d}$, $\left.{ }^{2} J_{\mathrm{CP}}=5.9, \mathrm{CH}_{2} \mathrm{O}\right), 62.8\left(\mathrm{~d},{ }^{2} J_{\mathrm{C}, \mathrm{P}}=8.9, \mathrm{C}(5)\right), 115.0\left(\mathrm{~d},{ }^{3} J_{\mathrm{C}, \mathrm{P}}=11.6\right.$, $\left.\mathrm{CH}_{\mathrm{Ph}}\right), 118.9\left(\mathrm{~s}, \mathrm{CH}_{\mathrm{Ar}}\right), 119.2\left(\mathrm{~s}, \mathrm{C}_{\mathrm{Ar}}\right), 119.7\left(\mathrm{~d}, J_{\mathrm{C}, \mathrm{P}}=1.4, \mathrm{CH}_{\mathrm{Ar}}\right)$, $119.8\left(\mathrm{~s}, \mathrm{C}_{\mathrm{Ar}}\right), 120.4\left(\mathrm{~s}, \mathrm{C}_{\mathrm{Ar}}\right), 120.7\left(\mathrm{~s}, \mathrm{C}_{\mathrm{Ar}}\right), 124.3\left(\mathrm{~s}, \mathrm{CH}_{\mathrm{Ar}}\right), 124.4$ $\left(\mathrm{s}, \mathrm{CH}_{\mathrm{Ar}}\right), 126.5\left(\mathrm{~s}, \mathrm{CH}_{\mathrm{Ar}}\right), 126.56\left(\mathrm{~s}, \mathrm{CH}_{\mathrm{Ar}}\right), 126.6\left(\mathrm{~s}, \mathrm{CH}_{\mathrm{Ar}}\right), 126.63$ $\left(\mathrm{s}, \mathrm{CH}_{\mathrm{Ar}}\right), 127.0\left(\mathrm{~s}, \mathrm{CH}_{\mathrm{Ar}}\right), 127.4\left(\mathrm{~s}, \mathrm{CH}_{\mathrm{Ar}}\right), 127.5\left(\mathrm{~s}, \mathrm{CH}_{\mathrm{Ar}}\right), 127.6$ $\left(\mathrm{s}, \mathrm{CH}_{\mathrm{Ar}}\right), 128.0\left(\mathrm{~s}, \mathrm{CH}_{\mathrm{Ar}}\right), 129.1\left(\mathrm{~s}, \mathrm{CH}_{\mathrm{Ar}}\right), 130.4$ (very br., $\left.\mathrm{CH}_{\mathrm{Ar}}\right)$, 131.8 (very br., $\left.\mathrm{CH}_{\mathrm{Ar}}\right), 133.1\left(\mathrm{~s}, \mathrm{CH}_{\mathrm{Ar}}\right), 133.3\left(\mathrm{~s}, \mathrm{CH}_{\mathrm{Ar}}\right), 134.5(\mathrm{~s}$, $\left.\mathrm{CH}_{\mathrm{Ar}}\right), 134.6\left(\mathrm{~s}, \mathrm{CH}_{\mathrm{Ar}}\right), 142.4\left(\mathrm{~s}, \mathrm{C}_{\mathrm{Ar}}\right), 142.7\left(\mathrm{~s}, \mathrm{C}_{\mathrm{Ar}}\right), 142.8(\mathrm{~d}$, $\left.J_{\mathrm{C}, \mathrm{P}}=2.1, \mathrm{C}_{\mathrm{Ar}}\right), 146.0\left(\mathrm{~d},{ }^{2} J_{\mathrm{C}, \mathrm{P}}=15.8, \mathrm{C}_{\mathrm{Ph}}\right) .{ }^{31} \mathrm{P} \mathrm{NMR}(242.9 \mathrm{MHz}$, $\left.\mathrm{C}_{6} \mathrm{D}_{6}, 27^{\circ} \mathrm{C}\right) \delta_{\mathrm{P}} \mathrm{ppm}: 118.3$.

$\left(S_{0}\right)$-4-(4-(10,15,20-Triphenylporphyrin-5-yl)phenoxy) dinaphtho[2,1-d:1',2'-f][1,3,2] dioxaphosphepine (5). Violet powder $(0.76 \mathrm{~g}$, yield $80 \%)$. [a $]_{\mathrm{D}}{ }^{22}=+188.4^{\circ}$ (c 1.0, toluene). Found, \%: C, 81.55; H, 4.30; N, 5.79. $\mathrm{C}_{64} \mathrm{H}_{41} \mathrm{~N}_{4} \mathrm{O}$ P. Calculated, \%: 
C, 81.34; H, 4.37; N, 5.93. Mass spectra (MALDI TOF/TOF) $\mathrm{m} / \mathrm{z}$ (\%): $945(100)\left([\mathrm{M}+\mathrm{H}]^{+}\right), 631(58)\left(\left[\mathrm{C}_{44} \mathrm{H}_{30} \mathrm{~N}_{4} \mathrm{O}+\mathrm{H}\right]^{+}\right) .{ }^{1} \mathrm{H}$ NMR $\left(600.13 \mathrm{MHz}, \mathrm{CDCl}_{3}, 25^{\circ} \mathrm{C}\right) \delta_{\mathrm{H}}$ ppm: -2.73 (br. s, $\left.2 \mathrm{H}, \mathrm{NH}\right), 7.34$ 7.39 (m, $2 \mathrm{H}, \mathrm{Ar}$ ), 7.49-7.55 (m, $4 \mathrm{H}, \mathrm{Ar}$ ), 7.61 (d, ${ }^{3} J_{\mathrm{H}, \mathrm{H}}=8.4$, $2 \mathrm{H}$, Ar.), 7.67 (d, $\left.{ }^{3} J_{\mathrm{H}, \mathrm{H}}=9.0,1 \mathrm{H}, \mathrm{Ar}.\right), 7.74\left(\mathrm{~d},{ }^{3} J_{\mathrm{H}, \mathrm{H}}=8.4,1 \mathrm{H}\right.$, Ar.), 7.77-7.83 (m, 9 H, Ar.), 8.01 (d, ${ }^{3} J_{\mathrm{H}, \mathrm{H}}=7.8,1 \mathrm{H}, \mathrm{Ar}$ ), 8.03 $\left(\mathrm{d},{ }^{3} J_{\mathrm{HH}}=7.2,1 \mathrm{H}, \mathrm{Ar}.\right), 8.08\left(\mathrm{~d},{ }^{3} J_{\mathrm{HH}}=9.0,1 \mathrm{H}, \mathrm{Ar}.\right), 8.11(\mathrm{~d}$, ${ }^{3} J_{\mathrm{H}, \mathrm{H}}=9.0,1 \mathrm{H}, \mathrm{Ar}$ ), $8.20\left(\mathrm{~d},{ }^{3} \mathrm{~J}_{\mathrm{H}, \mathrm{H}}=8.4,2 \mathrm{H}, \mathrm{Ar}\right.$ ), $8.25-8.28(\mathrm{~m}$, $6 \mathrm{H}, \mathrm{Ar}$ ), $8.86-8.91$ (m, $8 \mathrm{H}, \mathrm{Ar}.) .{ }^{13} \mathrm{C} \mathrm{NMR}\left(150.9 \mathrm{MHz}, \mathrm{CDCl}_{3}\right.$, $\left.25{ }^{\circ} \mathrm{C}\right) \delta_{\mathrm{C}} \mathrm{ppm}: 118.8\left(\mathrm{~d}, J_{\mathrm{C}, \mathrm{P}}=7.6, \mathrm{CH}_{\mathrm{Ar}}\right), 119.1\left(\mathrm{~s}, \mathrm{C}_{\mathrm{Ar}}\right), 120.26$ $\left(\mathrm{s}, \mathrm{C}_{\mathrm{Ar}}\right), 120.3\left(\mathrm{~s}, \mathrm{C}_{\mathrm{Ar}}\right), 121.8\left(\mathrm{~d}, J_{\mathrm{C}, \mathrm{P}}=1.4, \mathrm{CH}_{\mathrm{Ar}}\right), 121.9\left(\mathrm{~s}, \mathrm{CH}_{\mathrm{Ar}}\right)$, $123.0\left(\mathrm{~d}, J_{\mathrm{C}, \mathrm{P}}=2.5, \mathrm{C}_{\mathrm{Ar}}\right), 124.5\left(\mathrm{~d}, J_{\mathrm{C}, \mathrm{P}}=5.2, \mathrm{C}_{\mathrm{Ar}}\right), 125.2\left(\mathrm{~s}, \mathrm{CH}_{\mathrm{Ar}}\right)$, $125.4\left(\mathrm{~s}, \mathrm{CH}_{\mathrm{Ar}}\right), 126.4\left(\mathrm{~s}, \mathrm{CH}_{\mathrm{Ar}}\right), 126.5\left(\mathrm{~s}, \mathrm{CH}_{\mathrm{Ar}}\right), 126.72\left(\mathrm{~s}, \mathrm{CH}_{\mathrm{Ar}}\right)$, $126.73\left(\mathrm{~s}, \mathrm{CH}_{\mathrm{Ar}}\right), 127.1\left(\mathrm{~s}, \mathrm{CH}_{\mathrm{Ar}}\right), 127.2\left(\mathrm{~s}, \mathrm{CH}_{\mathrm{Ar}}\right), 127.8\left(\mathrm{~s}, \mathrm{CH}_{\mathrm{Ar}}\right)$, $128.48\left(\mathrm{~s}, \mathrm{CH}_{\mathrm{Ar}}\right), 128.5\left(\mathrm{~s}, \mathrm{CH}_{\mathrm{Ar}}\right), 130.1\left(\mathrm{~s}, \mathrm{CH}_{\mathrm{Ar}}\right), 131.2$ (very br., $\left.\mathrm{CH}_{\mathrm{Ar}}\right), 131.4\left(\mathrm{~s}, \mathrm{C}_{\mathrm{Ar}}\right), 131.8\left(\mathrm{~s}, \mathrm{C}_{\mathrm{Ar}}\right), 132.7\left(\mathrm{~d}, J_{\mathrm{C}, \mathrm{P}}=1.1, \mathrm{C}_{\mathrm{Ar}}\right)$, $133.0\left(\mathrm{dr}, J_{\mathrm{C}, \mathrm{P}}=1.2, \mathrm{C}_{\mathrm{Ar}}\right), 134.59\left(\mathrm{~s}, \mathrm{CH}_{\mathrm{Ar}}\right), 134.61\left(\mathrm{~s}, \mathrm{CH}_{\mathrm{Ar}}\right), 135.7$ $\left(\mathrm{s}, \mathrm{CH}_{\mathrm{Ar}}\right), 138.4\left(\mathrm{~s}, \mathrm{C}_{\mathrm{Ar}}\right), 142.2\left(\mathrm{~s}, \mathrm{C}_{\mathrm{Ar}}\right), 147.1\left(\mathrm{~s},{ }^{2} J_{\mathrm{C}, \mathrm{P}}=2.3, \mathrm{POC}\right)$, $147.8\left(\mathrm{~d},{ }^{2} J_{\mathrm{C}, \mathrm{P}}=4.7, \mathrm{POC}\right), 151.7\left(\mathrm{~d},{ }^{2} J_{\mathrm{C}, \mathrm{P}}=6.9, \mathrm{POC}\right) .{ }^{31} \mathrm{P} \mathrm{NMR}$ (242.9 $\left.\mathrm{MHz}, \mathrm{CDCl}_{3}, 25^{\circ} \mathrm{C}\right) \delta_{\mathrm{P}} \mathrm{ppm}: 143.3$.

\section{Catalytic Reactions}

Pd-catalyzed allylic sulfonylation of (E)-1,3-diphenylallyl acetate 6 with sodium p-toluene sulfinate. A solution of [Pd(allyl) $\mathrm{Cl}]_{2}(1.9 \mathrm{mg}, 0.005 \mathrm{mmol})$ and the appropriate ligand $(0.01 \mathrm{mmol}$ or $0.02 \mathrm{mmol})$ in THF $(1.5 \mathrm{ml})$ was stirred for $40 \mathrm{~min}$. $(E)-1,3-$ Diphenylallyl acetate $(0.05 \mathrm{ml}, 0.25 \mathrm{mmol})$ was added and the solution stirred for $15 \mathrm{~min}$, then sodium $p$-toluene sulfinate $(0.089 \mathrm{~g}$, $0.5 \mathrm{mmol}$ ) was added and the reaction mixture was stirred for a further $48 \mathrm{~h}$, quenched with brine $(3 \mathrm{ml})$ and extracted with THF $(3 \times 2 \mathrm{ml})$. The organic layer was washed with brine $(2 \times 2 \mathrm{ml})$ and dried over $\mathrm{MgSO}_{4}$. The solvent was evaporated at reduced pressure (40 Torr). Crystallization of the residue from $\mathrm{EtOH}$, followed by desiccation in vacuo (10 Torr, $12 \mathrm{~h}$ ), has given (E)-1,3-diphenyl-3-tosylprop1-ene $\mathbf{7 a}$ as white crystals. ${ }^{[7,71]}$ The enantiomeric excess of 7a was determined by HPLC (Daicel Chiralcel OD-H column, $\mathrm{C}_{6} \mathrm{H}_{14} / i$ $\mathrm{PrOH}=4 / 1,0.5 \mathrm{ml} / \mathrm{min}, 254 \mathrm{~nm}, t(R)=16.3 \mathrm{~min}, t(S)=18.5 \mathrm{~min})$.

$P d$-catalyzed allylic alkylation of (E)-1,3-diphenylallyl acetate 6 with dimethyl malonate. A solution of $[\mathrm{Pd}(\mathrm{allyl}) \mathrm{Cl}]_{2}(1.9$ $\mathrm{mg}, 0.005 \mathrm{mmol})$ and the appropriate ligand $(0.01 \mathrm{mmol}$ or 0.02 $\mathrm{mmol})$ in the appropriate solvent $(1.5 \mathrm{ml})$ was stirred for $40 \mathrm{~min}$. (E)-1,3-Diphenylallyl acetate $(0.05 \mathrm{ml}, 0.25 \mathrm{mmol})$ was added, and the solution was stirred for $15 \mathrm{~min}$. Dimethyl malonate $(0.05$ $\mathrm{ml}, 0.44 \mathrm{mmol})$, BSA $(0.11 \mathrm{ml}, 0.44 \mathrm{mmol})$ and potassium acetate $(0.002 \mathrm{~g})$ were added. The reaction mixture was stirred for 48 h, diluted with $\mathrm{CH}_{2} \mathrm{Cl}_{2}$ or THF $(2 \mathrm{ml})$ and filtered through a thin layer of silica gel. The filtrate was evaporated at reduced pressure (40 Torr) and dried in vacuo (10 Torr, $12 \mathrm{~h}$ ) affording a residue containing $(E)$-dimethyl 2-(1,3-diphenylallyl)malonate $\quad \mathbf{7 b}^{[72,73]}$ In order to evaluate $e e$ and conversion, the obtained residue was dissolved in an appropriate eluent mixture $(8 \mathrm{ml})$ and a sample was taken for HPLC analysis (Daicel Chiralcel OD-H column, $\mathrm{C}_{6} \mathrm{H}_{14} / i$ $\mathrm{PrOH}=99 / 1,0.3 \mathrm{ml} / \mathrm{min}, 254 \mathrm{~nm}, t(R)=28.0 \mathrm{~min}, t(S)=29.3 \mathrm{~min})$.

Pd-catalyzed allylic amination of (E)-1,3-diphenylallyl acetate 6 with pyrrolidine. A solution of $[\mathrm{Pd}(\text { allyl }) \mathrm{Cl}]_{2}(1.9 \mathrm{mg}$, $0.005 \mathrm{mmol})$ and the appropriate ligand $(0.01 \mathrm{mmol}$ or $0.02 \mathrm{mmol})$ in the appropriate solvent $(1.5 \mathrm{ml})$ was stirred for $40 \mathrm{~min}$. $(E)-1,3-$ Diphenylallyl acetate $(0.05 \mathrm{ml}, 0.25 \mathrm{mmol})$ was added and the solution was stirred for $15 \mathrm{~min}$, then freshly distilled pyrrolidine $(0.06 \mathrm{ml}, 0.75 \mathrm{mmol})$ was added. The reaction mixture was stirred for $48 \mathrm{~h}$, diluted with $\mathrm{CH}_{2} \mathrm{Cl}_{2}$ or THF $(2 \mathrm{ml})$ and filtered through a thin layer of silica gel. The filtrate was evaporated at reduced pressure (40 Torr) and dried in vacuo (10 Torr, $12 \mathrm{~h}$ ) affording a residue containing $(E)$-1-(1,3-diphenylallyl)pyrrolidine $7 \mathbf{c}^{[74,75]}$ In order to evaluate $e e$ and conversion, the obtained residue was dissolved in an appropriate eluent mixture $(8 \mathrm{ml})$ and a sample was taken for HPLC analysis (Daicel Chiralcel OD-H column, $\mathrm{C}_{6} \mathrm{H}_{14} / i$ -
$\mathrm{PrOH} / \mathrm{HN}(\mathrm{Et})_{2}=200 / 1 / 0.1,0.9 \mathrm{ml} / \mathrm{min}, 254 \mathrm{~nm}, t(R)=5.0 \mathrm{~min}$, $t(S)=6.1 \mathrm{~min})$.

Pd-catalyzed allylic alkylation of cinnamyl acetate 8 with ethyl 2-oxocyclohexane-1-carboxylate 9. A solution of [Pd(allyl) $\mathrm{Cl}]_{2}(1.9 \mathrm{mg}, 0.005 \mathrm{mmol})$ and the appropriate ligand $(0.01 \mathrm{mmol}$ or $0.02 \mathrm{mmol})$ in the appropriate solvent $(1.5 \mathrm{ml})$ was stirred for $40 \mathrm{~min}$. Cinnamyl acetate $(0.04 \mathrm{ml}, 0.25 \mathrm{mmol})$ was added, and the solution was stirred for $15 \mathrm{~min}$. Ethyl 2-oxocyclohexane-1carboxylate $(0.06 \mathrm{ml}, 0.375 \mathrm{mmol})$, BSA $(0.25 \mathrm{ml}, 1 \mathrm{mmol})$ and zinc acetate $(0.005 \mathrm{~g})$ were added. The reaction mixture was stirred for $48 \mathrm{~h}$, diluted with $\mathrm{CH}_{2} \mathrm{Cl}_{2}$ or toluene $(2 \mathrm{ml})$ and filtered through a thin layer of silica gel. The filtrate was evaporated at reduced pressure (40 Torr) and dried in vacuo (10 Torr, $12 \mathrm{~h}$ ) affording a residue containing ethyl 1-cinnamyl-2-oxocyclohexanecarboxylate $10{ }^{[53]}$ In order to evaluate $e e$ and conversion, the obtained residue was dissolved in an appropriate eluent mixture $(8 \mathrm{ml})$ and a sample was taken for HPLC analysis (Kromasil 5-CelluCoat, $\mathrm{C}_{6} \mathrm{H}_{14} / i$ $\mathrm{PrOH}=95 / 5,0.4 \mathrm{ml} / \mathrm{min}, 254 \mathrm{~nm}, t(R)=14.3 \mathrm{~min}, t(S)=16.4 \mathrm{~min})$.

Acknowledgements. We acknowledge the financial support from the Russian Science Foundation (Grant No. 14-1301383).

\section{References}

1. Brown J.M. In: Comprehensive Asymmetric Catalysis, Vol. 1 (Jacobsen E.N., Pfaltz A., Yamamoto H., Eds.), Springer: Berlin, 1999. pp. 121-182.

2. Ohkuma T., Kitamura M., Noyori R. In: Catalytic Asymmetric Synthesis, $2^{\text {nd }}$ Ed., (Ojima I., Ed.), New York: Wiley-VCH, 2000. pp. 1-110.

3. Trost B. M., Crawley M. L. Chem. Rev. 2003, 103, 2921-2943.

4. Blaser H.-U., Federsel H.-J. In: Asymmetric Catalysis on Industrial Scale: Challenges, Approaches, and Solutions, (Blaser H.-U., Federsel H.-J., Eds.), Weinheim: Wiley-VCH, 2010.

5. van Leeuwen P.W.N.M., Kamer P.C.J., Claver C., Pamies O., Dieguez M. Chem. Rev. 2011, 111, 2077-2118.

6. Beletskaya I.P., Ananikov V.P. Organometallics 2011, 30, 5-6.

7. Claver C., Pamies O., Dieguez M. In: Phosphorus Ligands in Asymmetric Catalysis, Vol. II (Börner A., Ed.), Weinheim: Wiley-VCH, 2008. pp. 507-528.

8. Falciola C.A., Alexakis A. Eur. J. Org. Chem. 2008, 37653780 .

9. Hargaden G.C., Guiry P.J. Chem. Rev. 2009, 109, 2505-2550.

10. Bini L., Muller C., Vogt D. Chem. Commun. 2010, 8325-8334.

11. Teichert J.F., Feringa B.L. Angew. Chem., Int. Ed. 2010, 49, 2486-2528.

12. Phosphorus(III) Ligands in Homogeneous Catalysis: Design and Synthesis (Kamer P.C.J., van Leeuwen P.W.N.M., Eds.), Chichester: Wiley-VCH, 2012.

13. Gavrilov K.N., Shiryaev A.A., Zheglov S.V., Gavrilov V.K., Groshkin N.N., Maksimova M.G., Volov A.N., Zamilatskov I.A. Tetrahedron 2014, 70, 616-624.

14. Wassenaar J., Reek J.N.H. Org. Biomol. Chem. 2011, 9, 17041713.

15. Tolstikov A.G., Khlebnikova T.B., Tolstikova O.V., Tolstikov G.A. Russ. Chem. Rev. 2003, 72, 803-822.

16. Dieguez M., Ruiz A., Claver C. Dalton Trans. 2003, 29572963.

17. Stanley L.M., Sibi M.P. Chiral Bisoxazoline Ligands. In: Privileged Chiral Ligands and Catalysts (Zhou Q.-L., Ed.), Weinheim: Wiley-VCH, 2011.

18. Gavrilov K.N., Zheglov S.V., Gavrilov V.K., Chuchelkin I.V., Novikov I.M., Shiryaev A.A., Volov A.N., Zamilatskov I.A. Tetrahedron: Asymmetry 2014, 25, 1116-1121.

19. Ansell J., Wills M. Chem. Soc. Rev. 2002, 31, 259-268. 
20. Alexakis A., Benhaim C. Eur. J. Org. Chem. 2002, 19, 32213236.

21. Molt O., Schrader T. Synthesis 2002, 2633-2670.

22. Reetz M.T., Mehler G., Meiswinkel A., Sell T. Tetrahedron Lett. 2002, 43, 7941-7943.

23. Dieguez M., Pamies O., Claver C. Tetrahedron: Asymmetry 2004, 15, 2113-2122.

24. Gavrilov K.N., Bondarev O.G., Polosukhin A.I. Russ. Chem. Rev. 2004, 73, 671-699.

25. Jerphagnon T., Renaud J.-L., Bruneau C. Tetrahedron: Asymmetry 2004, 15, 2101-2111.

26. Reetz M.T., Mehler G., Bondarev O. Chem. Commun. 2006, 2292-2294.

27. de Vries J.G., Lefort L. Chem. Eur. J. 2006, 12, 4722-4734.

28. Jagt R.B.C., Toullec P.Y. Geerdink D., de Vries J.G., Feringa B.L., Minnaard A.J. Angew. Chem., Int. Ed. 2006, 45, 27892791.

29. Mata Y., Pamies O., Dieguez M. Chem. Eur. J. 2007, 13, 3296 3304.

30. Swennenhuis B.H.G., Chen R., van Leeuwen P.W.N.M., de Vries J.G., Kamer P.C.J. Org. Lett. 2008, 10, 989-992.

31. Qiao X.-C., Zhu S.-F., Zhou Q.-L. Tetrahedron: Asymmetry 2009, 20, 1254-1261.

32. Crepy K.V.L., Imamoto T. Adv. Synth. Catal. 2003, 345, 79101.

33. Grabulosa A. P-Stereogenic Ligands in Enantioselective Catalysis; Cambridge: Royal Society of Chemistry, 2011.

34. Meeuwissen J., Reek J.N.H. Nat. Chem. 2010, 2, 615-621.

35. Carboni S., Gennari C., Pignataro L., Piarulli U. Dalton Trans. 2011, 40, 4355-4373.

36. Bellini R., van der Vlugt J.I., Reek J.N.H. Isr. J. Chem. 2012, 52, 613-629.

37. Raynal M., Ballester P., Vidal-Ferran A., van Leeuwen P.W.N.M. Chem. Soc. Rev. 2014, 43, 1660-1733.

38. Ohmatsu K., Ooi T. Tetrahedron Lett. 2015, 56, 2043-2048.

39. Slagt V.F., Roder M, Kamer P.C.J., van Leeuwen P.W.N.M., Reek J.N.H. J. Am. Chem. Soc. 2004, 126, 4056-4057.

40. Jiang X.-B., Lefort L., Goudriaan P.E., de Vries A.H.M., van Leeuwen P.W.N.M., de Vries J.G., Reek J.N.H. Angew. Chem. Int. Ed. 2006, 45, 1223-1227.

41. Xianq X.-B., van Leeuwen P.W.N.M., Reek J.N.H. Chem. Commun. 2007, 2287-2289.

42. Goudriaan P.E., Jang X.-B., Kuil M., Lemmens R., van Leeuwen P.W.N.M., Reek J.N.H. Eur. J. Org. Chem. 2008, 6079-6092.

43. Goudriaan P.E., Kuil M., Jiang X.-B., van Leeuwen P.W.N.M., Reek J.N.H. Dalton Trans., 2009, 1801-1805.

44. Fernandez-Perez H., Etayo P., Panossian A., Vidal-Ferran A. Chem. Rev. 2011, 111, 2119-2176.

45. McCarthy M., Guiry P.J. Tetrahedron 2001, 57, 3809-3844.

46. Graening T., Schmalz H.-G .Angew. Chem., Int. Ed. 2003, 42, 2580-2584.

47. Chapsal B.D., Ojima I. Org. Lett. 2006, 8, 1395-1398.

48. Lu Z., Ma S. Angew. Chem., Int. Ed. 2008, 47, 258-297.
49. Dieguez M., Pamies O. Acc. Chem. Res. 2010, 43, 312-322.

50. Lam F.L., Kwong F.Y., Chan A.S.C. Chem. Commun. 2010, 4649-4667.

51. Lafrance D., Bowles P., Leeman K., Rafka R. Org. Lett. 2011, 13, 2322-2325.

52. Nemoto T., Hamada Y. Tetrahedron 2011, 67, 667-687.

53. Nemoto T., Matsumoto T., Masuda T., Hitomi T., Hatano K., Hamada Y. J. Am. Chem. Soc. 2004, 126, 3690-3691.

54. Liu Y., Han S.-J., Liu W.-Bo, Stoltz B.M. Acc. Chem. Res. 2015, 48, 740-751.

55. Brunel J.M., Constantieux T., Buono G. J. Org. Chem. 1999, 64, 8940-8942.

56. Tsarev V.N., Lyubimov S.E., Shiryaev A.A., Zheglov S.V., Bondarev O.G., Davankov V.A., Kabro A.A., Moiseev S.K., Kalinin V.N., Gavrilov K.N. Eur. J. Org. Chem. 2004, 2214 2222.

57. Barta K., Hölscher M., Franciò G., Leitner W. Eur. J. Org. Chem. 2009, 4102-4116.

58. Kimura M., Uozumi Y. J. Org. Chem. 2007, 72, 707-714.

59. Gavrilov K.N., Zheglov S.V., Gavrilova M.N., Novikov I.M., Maksimova M.G., Groshkin N.N., Rastorguev E.A., Davankov V.A. Tetrahedron 2012, 68, 1581-1589.

60. Tolman C.A. Chem. Rev. 1977, 77, 313-348.

61. Polosukhin A.I., Kovalevskii A.I., Gavrilov K.N. Russ. J. Coord. Chem. 1999, 25, 758-761 (in Russ.).

62. Bunten K.A., Chen L., Fernandez A.L., Poe A. J. Coord. Chem. Rev. 2002, 233-234, 41-51.

63. Bilbrey J.A., Kazez A.H., Locklin J., Allen W.D. J. Comp. Chem. 2013, 34, 1189-1197.

64. Slagt V.F., van Leeuwen P.W.N.M., Reek J.N.H. Chem. Commun. 2003, 2474-2475.

65. Balasubramaniam E., Ramachandraiah G., Natarajan P., BiedCharreton C., Devynck J., Bediou F. J. Mater. Chem. 1995, 5, 625-629.

66. Annoni E., Pizzotti M., Ugo R., Quici S., Morotti T., Bruschi M., Mussini P. Eur. J. Inorg. Chem. 2005, 3857-3874.

67. Francio G., Arena C. G., Faraone F., Graiff C., Lanfranchi M., Tiripicchio A. Eur. J. Inorg. Chem. 1999, 1219-1227.

68. Auburn P.R., Mackenzie P.B., Bosnich B. J. Am. Chem. Soc. 1985, 107, 2033-2046.

69. Gavrilov K.N., Lyubimov S.E., Zheglov S.V., Benetsky E.B., Davankov V.A. J. Mol. Catal. A: Chemical 2005, 231, 255-260.

70. Seebach D., Devaquet E., Ernst A., Hayakawa M., Kuhnle F.N.M., Schweizer W.B., Weber B. Helv. Chim. Acta 1995, 78, 1636-1650.

71. Wolfe J.A., Hitchcock S.R. Tetrahedron: Asymmetry 2010, 21 , 2690-2695.

72. Breeden S., Wills M. J. Org. Chem. 1999, 64, 9735-9738.

73. Mei L.-Y., Yuan Z.-L., Shi M. Organometallics 2011, 30, 6466-6475.

74. Smyth D., Tye H., Eldred C., Alcock N.W., Wills M. J. Chem. Soc., Perkin Trans. 1 2001, 2840-2849.

75. Chen J., Lang F., Li D., Cun L., Zhu J., Deng J., Liao J. Tetrahedron: Asymmetry 2009, 20, 1953-1956. 